\title{
MORAVEC'S PARADOX: CONSIDERATION IN THE CONTEXT OF TWO BRAIN HEMISPHERE FUNCTIONS
}

\author{
Vadim S. Rotenberg* \\ Tel-Aviv University, Bat-Yam, Israel
}

\begin{abstract}
According to Moravec's paradox contrary to traditional assumptions, high-level reasoning requires very little computation compared to low-level sensorimotor skills that require enormous computational resources. For example, it is possible to make computers exhibit adult level performance on intelligence tests or playing checkers, but extremely difficult or impossible to give them the skills like perception or mobility. Several findings suggest that explanation of Moravec's paradox is related to the different functions of the human left and right hemispheres.
\end{abstract}

Key words: Cognitive reasoning; Sensorimotor skills; Brain hemispheres

\section{WHAT MEANS “MORAVEC'S PARADOX”}

The essence of the so-called Moravec's paradox (Moravec, 1998) is that high-level reasoning (abstract thinking, performing Intelligent Test, playing chess and checkers, using mathematics, engineering etc.), everything that seems to be hard for us and needs special skills requires much less computation and are more easy for reverse-engineer and programming, in comparison to low level sensory-motor skills like recognizing faces and voices, walking, moving around in space, distinguishing between simple objects. All these low level skills that are available for children actually require enormous computational resources for finding a relevant algorhythm and creating program, although in a real life this activity is performed almost automatically.

Moravec proposed the following explanation of this paradox. The intellectual skills that are available for computerization are the new one, they developed late, and had much less time for the natural selection in the course of evolution (only around 100000 years) to preserve design improvement and optimization, in comparison to more old skills.

Due to the long process of evolution that these older sensory-motor skills passed, we are already well adopted to them. Due to this long-lasting process of improvement, optimization and adaptation these skills seem to be easy for realization. However, it is only our subjective illusion: we became very familiar with these skills in the process of evolution but due to the long process of improvement and optimization they became very complicated for computerization. The relatively new skills like logical reasoning, solving mathematical tasks

${ }^{*}$ Correspondence to: Vadim Rotenberg, email: vadir@post.tau.ac.il

Received July 19, 2013; accepted August 10, 2013; Act Nerv Super (Praha) 55(3), 108-111. 
etc. on the first glance look out as more difficult because we are not familiar with them, but from the objective point of view they are less complicated due to the relatively short story of development. Moravec emphasized that the most difficult human skills to reverse engineer are those that are unconscious and it corresponds to the above mentioned explanation because consciousness represents phylogenetically very new skills. We are more aware of simple processes that don't work well than of complex ones that work flaw-less.

\section{ANOTHER APPROACH: THE ROLE OF BRAIN LATERALITY}

However, this explanation has some restrictions and weak points. The creativity in different domains belongs to the phylo- and ontogenetically most new skills. Only humans are owners of creative skills, and the brain area that is most responsible for these skills (right frontal lobe, Rotenberg, 2007) is the lastly matured brain structure (Saugstad, 1998). However it is very difficult and up to now even impossible to find an algorhythm of the process of creativity and to perform its computerization.

At the same time, it is interesting and necessary to take into consideration that for the creative subjects the process of creation by itself is almost effortless (Martindale, 1975): according to EEG data brain of creative subject is not physiologically activated while solving creative tasks, while solving logical tasks requires brain activation.

I suppose that explanation of these contradictions as well as the explanation of Moravec's paradox is related to the different functions of the humans left and right hemispheres in my interpretation (see Rotenberg, 1979; 1993; 2007). According to my concept, in the most general form the difference between the two strategies of thinking related to the left and right hemisphere is determined by the opposite modes of organizing the contextual relationships between objects and events, elements of information. Left-hemispheric formal logical thinking organizes any sign material (symbolic and iconic) as to create a strictly ordered and unambiguously understood monosemantic context. Its formation requires an active choice from the numerous real and potential connections between the multiform objects and phenomena of a few definite and restricted connections that would not cause internal contradictions and would facilitate an ordered analysis. Such a strategy of thinking makes it possible to build a pragmatically convenient but simplified model of reality. It is strong related to the function of speech because in the process of communication and collaboration it is very important to be able to present and accept the unambiguous and definitely understood monosemantic message.

In contrast, the function of the right hemisphere is a simultaneous capture of an infinite number of real connections and the formation of an integral but ambiguous polysemantic context due to this capture. In such a context the whole is not determined by its single components. All specific feature of the whole are determined by interconnections between these parts.

On the contrary, any concrete element of such a context bears a determining stamp of the whole. Every new experience became incorporated in this natural and holistic picture of the world. It is the function of the right frontal lobe. Due to this holistic view and this ability to integrate all interrelationships presented by the real world the right frontal lobe plays a key role in creativity.

At the same time, on the more low level, other right hemisphere structures are responsible for the sensory-motor integration in the world and this process also requires holistic grasping of all sensory information and the managing of the holistic motor behavior that corresponds to this information. Right hemisphere is also especially close related to the limbic system (Rotenberg, Weinberg, 1999) that controls body functions.

Thus, the right hemisphere displays on the one hand a system that determines a natural integration of the subject in the polydimensional world, and on the other hand is responsible for the creativity that also requires an integrative view on the world. It is easy for humans to 
adopt their right-hemispheric skills and to perform functions controlled by the right hemisphere just because these skills and functions are very native. However, their natural essence does not make them easy and available for analysis and reproduction. Quite opposite, just due to its simplicity for our natural every-day life they are very complicated for the computerization that is based on the quite opposite principles and requires left hemispheric skills. Science and intellectual games are more relevant for computerization.

\section{THE ARTIFICIAL ESSENCE OF MONOSEMANTIC SCIENTIFIC MODELS}

In comparison to the right hemisphere functions, the functions of the left hemisphere are in some way artificial. In order to build a monosemantic context, it is necessary to perform a disintegration of the holistic world, to select it in pieces and to choose definite and single relationships between them. Of course, it is obligate and unavoidable for analysis and its fixation as well as for communication but this obligation does not make it less artificial, from the natural direct point of view. Let us say, it is an obligated artifact. And the process of computation and of building algorhythms and systems like artificial intelligence is based on this function of the left hemisphere, on its ability to form a monosemantic context.

The formation of the monosemantic context and of the speech on the modern step of evolution modulates the real 1 world and makes it more structuralized and that's why these computative models correspond to the left hemisphere functions as a key to the lock.

The real world with all its natural polydimensional interrelationships we are integrated in by mean of our right hemisphere does not correspond to these artificial systems and its computation is not available.

\section{TWO TYPES OF CIVILIZATIONS}

The representatives of the Eastern civilization (India, China, Japan) are characterized by the domination of the right hemisphere skills while the representatives of the Western civilization are in general characterized by the domination of the left hemisphere due to the direction of education (Rotenberg \& Arshavsky, 1997). And it is not an occasion that for the representatives of the Eastern culture it is more easy to adopt themselves to the Western culture (Verma, 2013) than for the representatives of the Western civilization to be integrated in the Eastern society. I suppose that it has the same roots as Moravec's paradox. To find a key to the left hemispheric definite rules is more easy than to accept the right hemispheric skills if they are not developed enough or even suppressed in the process of maturation and education.

\section{REFERENCES}

Adam, W., Kazakov, D.V., \& Kazakov, Martindale C. (1975). What makes creative people different? Psychology Today, July, 44-48

Moravec, H. (1998). When will computer hardware match the human brain. Journal of Evolution and Technology, 1.

Rotenberg, V.S. (1979). Word and image: the problem of context. Dynamische Psychiatrie/ Dynamic Psychiatry, 59, 494-498.

Rotenberg V.S. (1993) Richness against freedom: two hemisphere functions and the problem of creativity. Europ. J. High Ability, 4, pp.11-19

Rotenberg, V.S. (2004) The ontogeny and asymmetry of the highest brain skills and the pathogenesis of schizophrenia. Behavioral and Brain Sciences, 27, 863-864.

Rotenberg, V.S. (2007) The asymmetry of the frontal lobe functions and the fundamental problems of mental health and psychotherapy. Dynamische Psychiatrie/Dynamic Psychiatry, 1-2, 51-68. 
Rotenberg, V.S., \& Arshavsky V.V. (1997) Right and left brain hemisphere activation in the representatives of two different cultures. Homeostasis in Health and Disease, 38, 49-57.

Rotenberg, V.S., \& Weinberg I. (1999) Human memory, cerebral hemispheres, and the limbic system: A new approach. Genetic, Social and General Psychology Monographs, 125, 45-70.

Saugstad L.F. (1998) Cerebral lateralization and rate of maturation. International Journal of Psychophysiology, 28, 37-62.

Verma J. (2013) An effort towards understanding the global Indian mind set. Dynamische Psychiatrie/ Dynamic Psychiatry, 1-2, 37-62. 